Chronic Obstructive Pulmonary Diseases: Journal of the COPD Foundation

\author{
Original Research
}

\title{
Pulmonary Predictors of Incident Diabetes in Smokers
}

Gregory L. Kinney, MPH, $\mathrm{PhD}^{1}$ Emma H. Baker, MRCP, $\mathrm{PhD}^{2}$ Oana L. Klein, MD, MS ${ }^{3}$

Jennifer L. Black-Shinn, MPH, $\mathrm{PhD}^{1}$ Emily S. Wan, $\mathrm{MD}^{4}$ Barry Make, MD ${ }^{5}$ Elizabeth Regan, MD, $\mathrm{PhD}^{5}$

Russell P. Bowler, MD, $\mathrm{PhD}^{5}$ Sharon M. Lutz, $\mathrm{PhD}^{6}$ Kendra A.Young, $\mathrm{PhD}^{1}$ Lindsey M. Duca, $\mathrm{MS}^{1}$

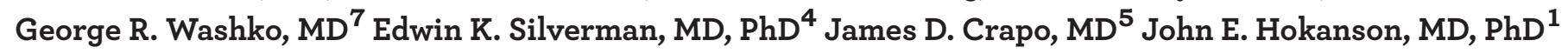

and the COPDGene Investigators

\section{Abstract}

Background: Diabetes mellitus and its complications are a large and increasing burden for health care worldwide. Reduced pulmonary function has been observed in diabetes (both type 1 and type 2), and this reduction is thought to occur prior to diagnosis. Other measures of pulmonary health are associated with diabetes, including lower exercise tolerance, greater dyspnea, lower quality of life (as measured by the St. George's Respiratory Questionaire [SGRQ]) and susceptibility to lung infection and these measures may also predate diabetes diagnosis.

Methods: We examined 7080 participants in the COPD Genetic Epidemiology (COPDGene) study who did not report diabetes at their baseline visit and who provided health status updates during 4.2 years of longitudinal follow-up (LFU). We used Cox proportional hazards modeling, censoring participants at final LFU contact, reported mortality or report of incident diabetes to model predictors of diabetes. These models were constructed using known risk factors as well as proposed markers related to pulmonary health, forced expiratory volume in 1 second (FEV1), forced vital capacity (FVC), FEV 1 FVC, respiratory exacerbations (RE), 6-minute walk distance (6MWD), pulmonary associated quality of life (as measured by the SGRQ), corticosteroid use, chronic bronchitis and dyspnea. Results: Over 21,519 person years of follow-up, 392 of 7080 participants reported incident diabetes which was associated with expected predictors; increased body mass index (BMI), high blood pressure, high cholesterol and current smoking status. Age, gender and accumulated smoking exposure were not associated with incident diabetes. Additionally, preserved ratio with impaired spirometry (PRISm) pattern pulmonary function, reduced 6MWD and any report of serious pulmonary events were associated with incident diabetes.

Conclusions: This cluster of pulmonary indicators may aid clinicians in identifying and treating patients with pre- or undiagnosed diabetes.

\begin{abstract}
Abbreviations: COPD Genetic Epidemiology, COPDGene; longitudinal follow-up, LFU; forced expiratory volume in 1 second, FEV $\mathbf{1}$; forced vital capacity, FVC; respiratory exacerbations, RE; 6-minute walk distance, 6MWD; body mass index, BMI; preserved ratio with impaired spirometry, PRISm; airway surface liquid, ASL; modified Medical Research Council, mMRC; acute exacerbation of COPD, AECOPD; hazard ratio, HR; confidence interval, $\mathbf{C I}$; odds ratio, OR

Funding Support: The project described was supported by Award Number R01 HL089897, Award Number R01 HL089856 and Award Number K01 HL125858 from the National Heart, Lung, and Blood Institute. The COPDGene ${ }^{\circledR}$ project is also supported by the COPD Foundation through contributions made to an Industry Advisory Board comprised of AstraZeneca, Boehringer Ingelheim, GlaxoSmithKline, Novartis, Pfizer, Siemens and Sunovion.

Date of Acceptance: May 27, 2016

Citation: Kinney GL, Baker EH, Klein OL, et al. Predictors of incident diabetes in smokers. Chronic Obstr Pulm Dis (Miami). 2016;3(4):739747. doi: http://dx.doi.org/10.15326/jcopdf.3.4.2016.0137
\end{abstract}

1 Department of Epidemiology, Colorado School of Public Health, University of Colorado Anschutz Medical Campus

2 St. George's, University of London, United Kingdom

3 Department of Medicine, University of California-San Francisco
4 Channing Division of Network Medicine, Brigham and Women's Hospital, Boston, Massachusetts

5 National Jewish Health and University of Colorado-Denver

6 Department of Biostatistics, Colorado School of Public Health, University of Colorado Anschutz Medical Campus 
7 Brigham and Women's Hospital and Harvard Medical School, Boston, Massachusetts

\section{Address correspondence to:}

Gregory L Kinney, MPH, PhD

Department of Epidemiology

Colorado School of Public Health

University of Colorado Denver

13001 East 17th Avenue

Room W3141A, Campus Box B-119

Aurora, CO 80045

Telephone: 303-724-4437

Email: Greg.Kinney@UCDenver.edu

\section{Keywords:}

chronic obstructive pulmonary disease; COPD; diabetes; pulmonary predictors

\section{Background}

Diabetes and its complications are a large and increasing burden for health care worldwide. Reduced pulmonary function has been observed in type 2 diabetes $^{1-5}$ and this reduction is thought to occur prior to diagnosis. ${ }^{6-9}$ Other measures of pulmonary health are associated with diabetes, including lower exercise tolerance, ${ }^{10}$ greater dyspnea and lower pulmonary associated quality of life (as measured by the St. George's Respiratory Questionaire [SGRQ] $)^{11}$ and these measures may also predate diabetes diagnosis. Hyperglycemia has been shown to increase glucose in airway surface liquid (ASL) ${ }^{12}$ promoting bacterial growth ${ }^{13}$ which may lead to increased pulmonary associated adverse events. ${ }^{14,15}$ These events can result in emergency room visits or hospitalizations and poor outcomes of those hospitalizations. ${ }^{16}$ Hyperglycemia can also reduce the effectiveness of the innate immune system of the lung by reducing surfactant protein- ${ }^{17}$ which may be associated with lung infection and reduced diffusing lung capacity. ${ }^{18,19}$ Smoking has also been shown to be an independent predictor of type 2 diabetes $^{20}$ and quitting smoking has been shown to reduce the risk of metabolic syndrome. ${ }^{21}$ Pulmonary risk factors beyond smoking such as reduced pulmonary function, reduced exercise capacity, increased dyspnea and increased propensity for lung infection may be associated with the development of diabetes.

This study used data collected at the baseline visit and during longitudinal follow-up of a large cohort of smokers (>10 pack years smoking history) from the Genetic Epidemiology of Chronic Obstructive Pulmonary Disease (COPDGene) study to investigate factors preceding diabetes diagnosis. Known diabetes risk factors include obesity, male gender, increased age, hypertension, high cholesterol and steroid use and potential diabetes risk factors include reduced measures of pulmonary function (forced expiratory volume in 1 second $\left[\mathrm{FEV}_{1}\right]$, forced vital capacity [FVC] and their ratio), reduced 6-minute walk distance (6MWD), poor pulmonary associated quality of life measured by SGRQ, increased modified Medical Research Council (mMRC) dyspnea scale, chronic bronchitis, chronic obstructive pulmonary disease (COPD) and reported respiratory infection.

\section{Methods}

The COPDGene study has been described in detail

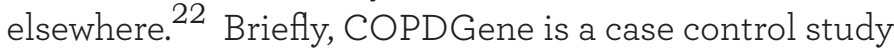
designed to identify genetic risk factors for COPD in a biracial population of non-Hispanic white $(\sim 2 / 3)$ and African American ( 1/3) smokers (current and former) with at least 10 pack years history of cigarette use. COPDGene participants were between 45 and 80 years old at initial contact, spanning COPD disease severity as measured by the Global initiative for chronic Obstructive Lung Disease (GOLD) criteria $^{23}$ and include 10,192 participants from 21 clinical centers. COPDGene includes a longitudinal follow-up (LFU) component that collects data on participants once every 6 months using telephony and web-based surveys, ${ }^{24}$ this study uses data from the 21/October/2013 LFU dataset. This study presents results from the baseline visit and follow-up surveys capturing events and new comorbidities in 7080 participants who responded for each of the analysis variables and who did not report past physician diagnosis of diabetes or diabetes-associated medications (biguanides, thiazolidinediones and other drugs used to treat type 2 diabetes) at the baseline visit. Follow-up surveys in this group were queried for a new physician report of diabetes using the question "Have you been diagnosed with diabetes by a doctor?" and a new report of diabetes was considered an event (incident diabetes diagnosis) for this analysis.

Pulmonary function, $\mathrm{FEV}_{1}, \mathrm{FVC}$ and $\mathrm{FEV}_{1} / \mathrm{FVC}$, were measured post-bronchodilator (albuterol) using the ndd EasyOne $^{\mathrm{TM}}$ Spirometer and all tests were performed according to guidelines published by the American Thoracic Society. ${ }^{25}$ FEV $_{1 \%}$ predicted and FVC\% predicted were used in models that did not include sex or ethnicity as these covariates are included in the sex and race-specific prediction equations. ${ }^{26}$ Quality of life was measured using the SGRQ which was obtained through participant self-report and overall and sub-scores were calculated using the appropriate standard protocol. ${ }^{27}$ The mMRC scale was obtained from the COPDGene 
questionnaire. Smoking exposure (current versus former), smoking intensity (pack years), medication use, high cholesterol and high blood pressure were obtained through participant self-report.

Respiratory exacerbations in COPD patients are referred to as acute exacerbation of COPD (AECOPD) and represents a worsening of symptoms of COPD that require alteration of medications or hospitalization. As AECOPD is specific to patients with COPD we will use the more general term respiratory exacerbations to refer to lung infections in participants without COPD as well as AECOPD in participants with COPD. Respiratory exacerbations were captured as part of the telephony interview using a series of questions capturing both changes in symptomatology and management change;

- "Since we last talked have you had an episode of

increased cough and phlegm or shortness of breath, which lasted 48 hours or more?

- Did you receive a new antibiotic?

- Did you receive any steroids either by pill or injection?

- Did you go urgently to your doctor's office?

- Did you go to an emergency room?

- Were you hospitalized?"

Respiratory exacerbation was recorded as Yes if the participant answered positively to receiving an antibiotic, steroid, urgent doctor's visit, emergency room visit or hospitalization after reporting changes in symptomatology at any LFU contact for this analysis.

Statistical analyses were performed using the SAS system version 9.3 (Copyright (c) 2002-2008 by SAS Institute Inc., Cary, North Carolina). We analyzed categorical data using $\chi^{2}$ test for overall effects and by category using Kruskal-Wallis and continuous data using $t$ tests (for normally distributed data) and transformed non-normally distributed data as appropriate and we considered a two-sided $p$ value of $\alpha<0.05$ to be statistically significant. Models were constructed using Cox Proportional Hazards models with time to event recorded as the time from the baseline visit until a physician diagnosis of diabetes, most recent contact via telephony or censoring due to all-cause mortality reported at the time of analysis. As a first step, a base model of incident diabetes diagnosis including known risk factors was fitted (sex, age, body mass index [BMI], smoking exposure, high cholesterol and high blood pressure) and these variables were retained in subsequent models. Additional pulmonary factors were added individually to this model to assess model improvement (FEV $1 \%, F V C \%, F_{1}$ FVVC, SAE, chronic bronchitis, 6MWD, mMRC dyspnea score, corticosteroid use and GOLD category). Model fit was assessed comparing the reduction in -2 Log Likelihood with the addition of a pulmonary covariate distributed as chi-square with degrees of freedom contingent on the variable included.

Individuals who do not meet the GOLD criteria for COPD $\left(\mathrm{FEV}_{1} / \mathrm{FVC}<0.7\right)$ but who have impaired spirometry $\left(\mathrm{FEV}_{1}<80 \%\right)$ are referred to as having preserved ratio with impaired spirometry (PRISm). ${ }^{28}$ All participants were placed into one of 3 categories; COPD (GOLD I-IV), PRISm or Control which includes all participants with neither COPD nor PRISm. FEV ${ }_{1} \%$ and FVC\% are strongly collinear (Pearson Rho=0.79, $p>=0.0001)$ and diabetes has been shown to have a stronger effect on $\mathrm{FVC} \%$ than on $\mathrm{FEV}_{1} \% .{ }^{5,11}$ We chose to exclude both $\mathrm{FEV}_{1} \%$ and $\mathrm{FVC} \%$ from the stepwise selection that was used to assess the most parsimonious model of incident diabetes and instead undertook stepwise models including either $\mathrm{FVC} \%$ or $\mathrm{FEV}_{1} \%$ separately.

\section{Results}

Incident diabetes was reported in 392 of 7080 participants during 21,519 person years of followup (mean follow-up time, 4.2 years). A total of 251 participants died and 15 participants' died after a report of incident diabetes. These participants follow-up time was truncated at their report of diabetes diagnosis. At the baseline visit, participants' who went on to develop diabetes were more likely to be current smokers ( $p=0.02)$, had higher BMI and increased high blood pressure and high cholesterol (all comparisons $p<0.0001$ ), but were not different by gender, pack years of smoking history or age [Table 1]. Participants classified as PRISm were observed to have higher incidence of diabetes diagnosis [Figure 1].

The base survival model of incident diabetes diagnosis included gender, age, smoking status, cumulative smoking exposure (pack years), high blood pressure, high cholesterol and BMI and this multivariable model agreed with the cross sectional results indicating that neither gender $(p=0.5)$, cumulative smoking exposure $(p=0.4)$ nor age (0.2) were significant predictors of diabetes [Table 2]. Subsequent models included these factors as potential confounders and precision variables. Significant predictors of incident diabetes diagnosis in the base model included current smoking status (hazard ratio $[\mathrm{HR}]=1.9$, 95\% confidence interval [CI] 1.5-2.4, $p$ 
Table 1. Characteristics of the COPDGene Cohort at Baseline Visit

\begin{tabular}{l|rrrr} 
& $\begin{array}{c}\text { Incident } \\
\text { Diabetes } \\
(\mathrm{n}=392)\end{array}$ & $\begin{array}{c}\text { Controls } \\
(\mathrm{n}=6688)\end{array}$ & \multicolumn{1}{c}{$p$} \\
\hline Age (SD) & $60.2 \pm 9.0$ & $60.3 \pm 9.0$ & 0.9 \\
\hline Pack Years Smoking (SD) & $46.0 \pm 26.2$ & $43.4 \pm 23.8$ & 0.05 \\
\hline Current Smoking (\%yes) & 0.54 & 0.47 & 0.02 \\
\hline Gender (\%m) & 0.49 & 0.50 & 0.8 \\
\hline BMI & $32.0 \pm 6.9$ & $28.2 \pm 5.9$ & $<0.0001$ \\
\hline High Blood Pressure (\%yes) & 0.53 & 0.37 & $<0.0002$ \\
\hline High Cholesterol (\%yes) & 0.48 & 0.38 & $<0.0003$ \\
\hline
\end{tabular}

Table 1 shows univariate comparisons of known risk factors for diabetes by incident diabetes status . BMI=body mass index; $\mathrm{SD}=$ standard deviation

\section{Figure 1. Incident Diabetes by GOLD Classification of COPD}

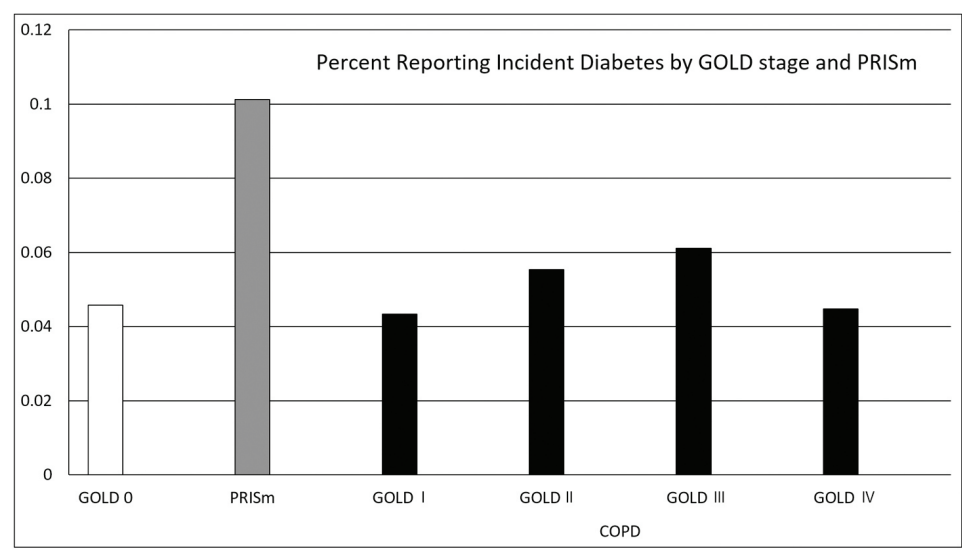

The $\mathrm{Y}$ axis indicates the percentage of participants at each GOLD stage reporting incident diabetes. GOLD $=$ Global initiative for chronic Obstructive Lung Disease PRISm=preserved ratio with impaired spirometry

\section{Table 2. Base Survival Model of Incident Diabetes}

\begin{tabular}{|c|c|c|c|}
\hline & $\begin{array}{c}\text { Hazard } \\
\text { Ratio }\end{array}$ & $\begin{array}{c}95 \% \\
\text { Confidence } \\
\text { Limits }\end{array}$ & $p$ \\
\hline Age (10 years) & 0.97 & $0.85-1.12$ & 0.7 \\
\hline ATS Pack Years (10 years) & 1.02 & $0.98-1.07$ & 0.3 \\
\hline Current Smoking (yes) & 1.91 & $1.52-2.39$ & $<0.0001$ \\
\hline Gender (m) & 1.07 & $0.87-1.31$ & 0.5 \\
\hline BMI (1 unit change) & 1.09 & $1.07-1.10$ & $<0.0001$ \\
\hline High Blood Pressure (yes) & 1.44 & $1.16-1.78$ & 0.0009 \\
\hline High Cholesterol (yes) & 1.33 & $1.08-1.64$ & 0.008 \\
\hline
\end{tabular}

Table 2 shows a Cox Proportional Hazards model including the known risk factors for incident diabetes collected by COPDGene. $p$-values $<0.05$ have been highlighted in yellow.
$<0.0001)$, BMI (HR=1.09, 95\% C.I. $1.07-1.10$ for 1 unit difference), high blood pressure (HR=1.4, 95\% CI 1.21.8, $p=0.0009)$ and high cholesterol $(\mathrm{HR}=1.3,95 \% \mathrm{CI}$ $1.1-1.6, p=0.0081$ ).

Both $\mathrm{FEV}_{1} \%$ and $\mathrm{FVC} \%$ were significantly associated with incident diabetes when added to the base model after controlling for other known risk factors ( $p=0.0004$ and $<0.0001$ respectively) though their ratio was not [Table 3]. Other pulmonary markers were also associated with incident diabetes; respiratory exacerbation during the follow-up period (HR 1.62, 95\% CI 1.23-2.08), lower 6MWD (HR 0.93, 95\% CI 0.91-0.96), corticosteroid use at baseline both oral (HR 1.80, 95\% CI 1.03-3.15) and inhaled (HR 1.81, 95\% CI 1.28-2.55), and PRISm pattern of lung function (HR 1.62, 95\% CI 1.46-2.61). Neither chronic bronchitis (HR 1.10, 95\% CI 0.85-1.42), nor COPD (HR 1.21, 95\% CI 0.95-1.55) were associated with incident diabetes in these models.

As this study represents only current and former smokers and over represents individuals with COPD compared to the general population, we explored respiratory exacerbation across GOLD stages and found that respiratory exacerbation occurred during the 4.2 years of follow-up at all levels of obstructive disease and also occurred in participants without COPD [Figure 2]. Participants with incident diabetes were at higher risk for respiratory exacerbation if they were PRISm $(22 \%$ versus $10 \%, p<0.0001)$ or GOLD $0(14 \%$ versus $5 \%, p=0.0006)$. Participants with COPD showed a significant increase in AECOPD only in GOLD III (45\% versus $31 \%, p=0.04$ ).

The most parsimonious model of incident diabetes assessed using stepwise selection of all pulmonary variables is presented in Table 4 . In addition to the base model of known risk factors, respiratory exacerbation ( $\mathrm{HR}=1.5,95 \%$ CI 1.2-2.0, $p=0.0009)$, reduced 6MWD $(-100$ feet; HR=0.94, 95\% CI 0.91-0.97, $p<0.0001)$ and PRISm (PRISm compared to GOLD 0; HR $1.7,95 \%$ CI 1.3-2.2, $p=0.0003$ ) were selected into the model. COPD (GOLD stage I-IV) was not significantly associated with incident diabetes ( $\mathrm{HR}=0.95,95 \%$ CI $0.7-1.2, p=0.7$ ) nor were the other potential markers measured as part of the COPDGene study.

\section{Discussion}

We observe, in this longitudinal study of current and former smokers, that markers of pulmonary health, beyond those measured using pulmonary function tests, are predictive of incident diabetes. Reduced 6MWD, 
respiratory exacerbation and PRISm were found to be associated with incident diabetes after controlling for known risk factors. While $\mathrm{FEV}_{1}$ and FVC have been shown to be associated with incident diabetes in other
Table 3. Univariate Associations Between Proposed Pulmonary Markers and Incident Diabetes

\begin{tabular}{|c|c|c|c|c|}
\hline & Estimate & Hazard Ratio & $95 \% \mathrm{Cl}$ & $p$ \\
\hline FEV $_{1} \%(10 \%$ change $)$ & -0.008 & 0.92 & $0.88-0.97$ & 0.0004 \\
\hline FVC\% (10\% change) & -0.014 & 0.87 & $0.82-0.93$ & $<0.0001$ \\
\hline FEV $_{1} /$ FVC (0.1 unit change) & -0.32 & 0.97 & $0.90-1.04$ & 0.4 \\
\hline Serious Pulmonary Event & 0.48 & 1.62 & $1.23-2.08$ & 0.0002 \\
\hline Chronic Bronchitis & 0.09 & 1.10 & $0.85-1.42$ & 0.48 \\
\hline Distance Walked (100 feet) & -0.0007 & 0.93 & $0.91-0.96$ & $<0.0001$ \\
\hline mMRC Dyspnea Score (0 vs 1 ) & 0.26 & 1.30 & $0.95-1.79$ & \multirow{4}{*}{0.0006} \\
\hline mMRC Dyspnea Score (0 vs 2 & 0.43 & 1.53 & $1.11-2.11$ & \\
\hline mMRC Dyspnea Score (0 vs 3) & 0.48 & 1.61 & $1.20-2.15$ & \\
\hline mMRC Dyspnea Score (0 vs 4) & 0.67 & 1.96 & $1.40-2.75$ & \\
\hline Oral Corticosteroid Use & 0.059 & 1.80 & $1.03-3.15$ & 0.04 \\
\hline Inhaled Corticosteroid Use & 0.59 & 1.81 & $1.28-2.55$ & 0.0008 \\
\hline PRISm & 0.67 & 1.95 & $1.46-2.61$ & \multirow{2}{*}{$<0.0001$} \\
\hline GOLD I-IV & 0.19 & 1.21 & $0.95-1.55$ & \\
\hline
\end{tabular}

Table 3 shows 10 models that include all variables from Model 1 (Table 2) plus each pulmonary associated variable in a separate model. $\mathrm{P}$ reported is for the association between each marker and incident diabetes. $p$-values $<0.05$ have been highlighted in yellow.

$\mathrm{CI}=$ confidence interval; $\mathrm{FEV}_{1}=$ forced expiratory volume in 1 second; $\mathrm{FVC}=$ forced vital capacity; mMRC=modified Medical Research Council; PRISm=preserved ratio with impaired spirometry; $\mathrm{GOLD}=\mathrm{Global}$ initiative for chronic Obstructive Lung Disease populations, these studies were not able to assess other measures of pulmonary health such as 6MWD, treatment for COPD with corticosteroids, dyspnea, respiratory exacerbation or specific types of lung disease

\section{Figure 2. Serious Pulmonary Events by Diabetes Status}

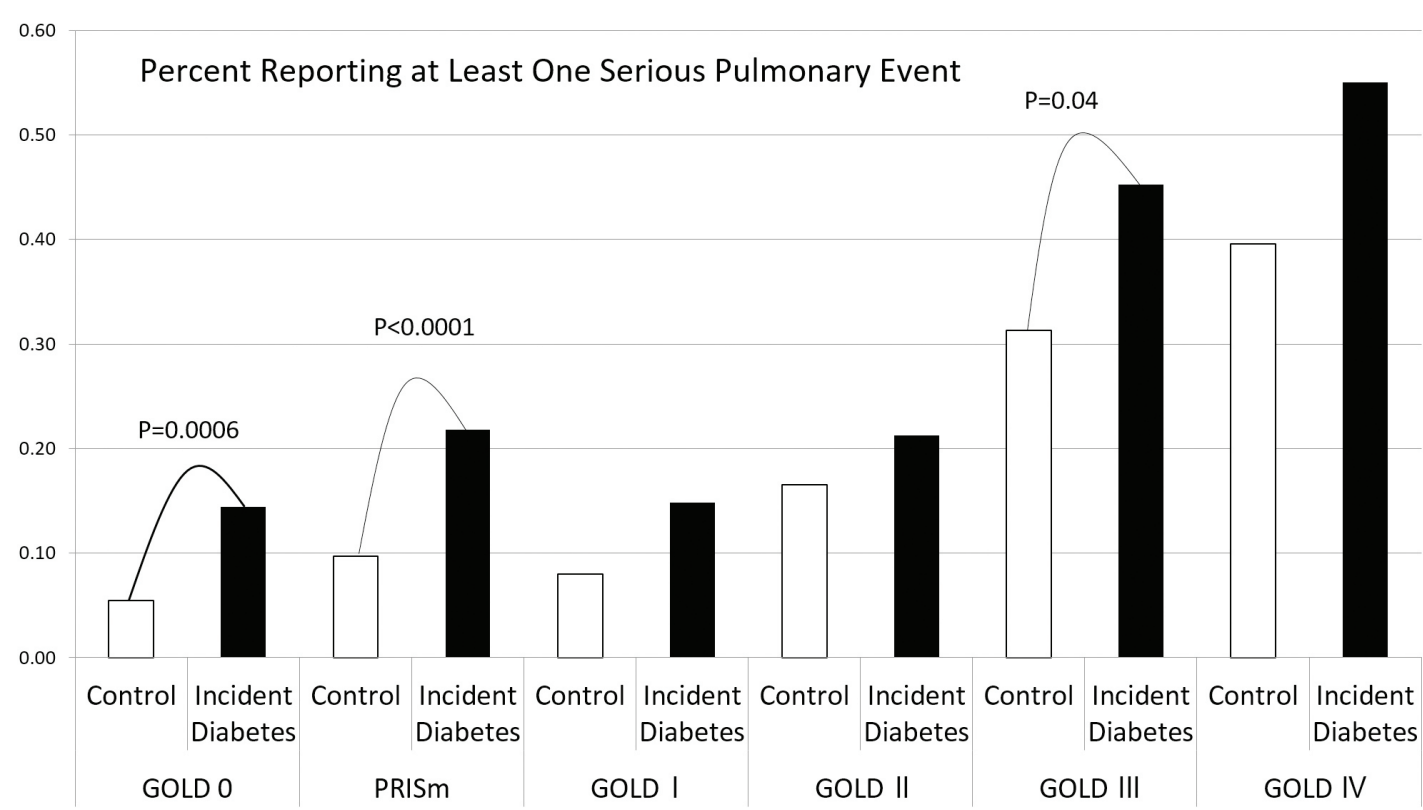

Figure 2 shows the percentage of participants reporting at least one serious pulmonary event (SPE)/acute exacerbation of COPD over the follow-up period (average 4.2 years) of the study. 


\section{Table 4. Final Survival Model of Incident Diabetes Including Pulmonary Measures}

\begin{tabular}{l|rrr}
\hline & $\begin{array}{c}\text { Hazard } \\
\text { Ratio }\end{array}$ & \multicolumn{1}{c}{$95 \% \mathrm{Cl}$} & \multicolumn{1}{c}{$p$} \\
\hline Age (10 years) & 0.97 & $0.8-1.1$ & 0.7 \\
\hline ATS Pack Years (10 years) & 1.0 & $0.96-1.05$ & 0.8 \\
\hline Current Smoking (yes) & 1.8 & $1.4-2.3$ & $<0.0001$ \\
\hline Gender (m) & 1.2 & $0.9-1.5$ & 0.07 \\
\hline BMI (1 unit change) & 1.1 & $1.1-1.1$ & $<0.0001$ \\
\hline High Blood Pressure (yes) & 1.3 & $1.1-1.7$ & 0.006 \\
\hline High Cholesterol (yes) & 1.4 & $1.1-1.7$ & 0.004 \\
\hline Exacerbation (yes) & 1.5 & $1.2-2.0$ & 0.0009 \\
\hline 6 MWD (100 feet) & 0.94 & $0.91-0.97$ & $<0.0001$ \\
\hline PRISm (versus GOLD 0) & 1.7 & $1.3-2.2$ & 0.0003 \\
\hline GOLD I-IV(versus GOLD 0) & 0.95 & $0.7-1.2$ & 0.7 \\
\hline
\end{tabular}

Table 4 shows the results of stepwise selection including Model 1 and selecting from $\mathrm{FEV}_{1} / \mathrm{FVC}$, SAE, chronic bronchitis, 6MWD, mMRC Dyspnea Score, corticosteroid use and GOLD classification. This selection was repeated adding $\mathrm{FEV}_{1}$ or $\mathrm{FVC}$ and the resulting models included identical variables. Neither FEV 1 nor FVC were selected into this model. $p$-values $<0.05$ have been highlighted in yellow. ATS=American Thoracic Society; BMI=body mass index; $6 \mathrm{MWD}=6$-minute walk distance; $\mathrm{PRISm}=$ preserved ratio with impaired spirometry; GOLD=Global initiative for chronic Obstructive Lung Disease; $\mathrm{FEV}_{1}=$ forced expiratory volume in 1 second;

$\mathrm{FVC}=$ forced vital capacity; $\mathrm{mMRC}=$ modified Medical Research Council

respiratory exacerbation. PRISm pattern pulmonary function is associated with obesity and obesity is on the metabolic pathway leading to type 2 diabetes. This is likely reflected in the association between PRISm and incident diabetes even after controlling for BMI though it should be noted that including the PRISm comparison into the model with BMI does not alter the effect of BMI on incident diabetes. BMI is known to be a poor marker of total adiposity and body composition making its use difficult to interpret precisely as a predictor of incident diabetes.

The lack of association between COPD and incident diabetes may be partially explained by the strong effect of COPD on 6MWD. A potential effect of pre or undiagnosed diabetes on the lungs is through increased blood glucose and glucose non-enzymatically binding with collagen and elastin in lung parenchyma resulting in reduced lung elasticity and reduced 6MWD due to restrictions in airflow. Therefore, obstructive pattern lung disease may not be an important, independent predictor of future diabetes once the result of that obstruction, measured by reduced 6MWD, is accounted for. This observation would also help explain why dyspnea is a strong predictor of incident diabetes on its own but no longer predictive after controlling for PRISm pattern lung disease as dyspnea may measure a reaction to sub-optimal inflation in pre or undiagnosed diabetes. This would also hold true if increased glucose affected the innate immune system of the lung, increasing the risk of lung infection and subsequent functional degradation as hyperglycemia is known to be associated with other types of infection. Chronic bronchitis is not associated with incident diabetes but respiratory exacerbation is, after controlling for known risk factors, 6MWD and PRISm. This suggests that smokers without COPD may be at increased risk for viral or bacterial lung infection.

We observed a known pattern of factors predicting pre or undiagnosed diabetes in these adult current and former smokers where age, gender, current smoking status, BMI, high blood pressure and lipid abnormalities were predictive of diabetes diagnosis over our longitudinal follow-up and remained significant with the addition of novel pulmonary-associated markers.

We hypothesized that individuals with pre or unrecognized diabetes were at increased risk for lung infection and that in the context of current and former heavy smoking that risk could be recognized as exacerbations of COPD or respiratory exacerbation in participants who do not have COPD. Surfactant-D is a member of the innate immune system found in the lungs which has been shown to be reduced in obesity and type 2 diabetes. ${ }^{29}$ Surfactant-D has been shown to be reduced in smokers ${ }^{30}$ and serum surfactant-D has been shown to be associated with exacerbations of COPD potentially due to increased lung permeability. ${ }^{31}$ Recent studies have shown that high blood glucose also affects the innate immune system by reducing the activity of beta-defensins which act against viral infection but also act against bacterial and fungal infection and are observed playing a protective role in the lungs. ${ }^{32}$ Kiselar et al observed laboratory evidence that hyperglycemia increases the production of methylgloxal and glyoxal which irreversibly adduct with human $\beta$-defensin-2, reducing its antimicrobial function ${ }^{33}$ making infection more likely during hyperglycemia. Hamilton et al reported data from the Freemantle Heart Study showing that participants with type 2 diabetes were at double the risk for hospitalization due to any infection compared to controls without diabetes and that the most common reason for hospitalization was pneumonia $(49.2 \%){ }^{34}$ A recent, large scale population based study using the Danish National Registry showed an association 
between antibiotic use and type 2 diabetes where antibiotic prescriptions were more likely to be filled in those with type 2 diabetes compared to controls (odds ratio [OR] 1.53, 95\% CI 1.50-1.55) and this observation was found both 5 years prior to diagnosis and 5 years post diagnosis with diabetes. ${ }^{35}$ Our study found that serious pulmonary events (SPE)/AECOPD were significant predictors of incident diabetes lending weight to this biological pathway.

Potential weaknesses of this study include the use of baseline measurement of known predictors that could not be assessed during the LFU as this followup protocol included telephony or web contact only. Future follow-up visits in the COPDGene cohort will collect these predictors and assess their change over time. Diabetes diagnosis is self-reported in COPDGene and has not been evaluated using known biomarkers such as fasting glucose, oral glucose tolerance test or glycosylated hemoglobin ( $\mathrm{HbA} 1 \mathrm{c})$. Baseline diabetes status was assessed using both self-report and recorded medication use and all participants reporting either were excluded from this incident study. Diabetes type was also not differentiated in COPDGene though the age structure of the study suggests that incident type 1 diabetes would be rare and all participants with existing diabetes were excluded from this incident study. Future studies of diabetes in COPDGene should assess diabetes status using gold standard biomarker measurement. The observed differences in SPE in participants without COPD (GOLD 0 and PRISm) may be due to a diabetes diagnosis that occurred because of health care contact resulting from the SPE itself. Evaluation of participant hospitalizations is not currently performed as a part of the COPDGene study.

The results of this study are of particular interest to clinicians as they identify new markers of pre or undiagnosed diabetes. SPEs, especially in current or former smokers without COPD (GOLD 0 and PRISm), may represent an early shift in a patient's glycemic control that affects susceptibility to lung infection. We have shown that SPEs are independently associated with incident diabetes in participants with PRISm, those with no functional impairment (GOLD 0) and those with COPD [Figure 2]. This may represent a population of current and former smokers preferentially affected by reduced innate immunity in their lungs due to poor glycemic control.

\section{Conclusions}

SPEs, reduced exercise capacity as measured by 6MWD and the PRISm pattern lung function impairment are significant, previously unrecognized predictors of the development of diabetes among current and former heavy smokers. This cluster of indicators may aid clinicians in identifying and treating patients with pre- or undiagnosed diabetes. Future studies should ascertain whether treatment for hyperglycemia and insulin resistance can reduce the occurrence of lung infection in this population.

\section{Acknowledgements}

The authors would like to express their gratitude to the administrators and DCC of the COPDGene Study. The content of this work is solely the responsibility of the authors and does not necessarily represent the official views of the National Heart, Lung and Blood Institutes or the National Institutes of Health.

\section{Declaration of Interest}

Gregory L. Kinney, Oana L. Klein, Jennifer L. BlackShinn, Emily S. Wan, Sharon M. Lutz, Elizabeth Regan, Russell P. Bowler, Kendra A. Young, Lindsey M. Duca, George R. Washko, James D. Crapo and John E. Hokanson have no conflicts to disclose. Emma H. Baker is joint holder of an MRC industry collaboration award with Astra Zeneca and principal investigator at St. George's for CRN portfolio adopted studies for Sirocco (Astra Zeneca), Ideal (GlaxoSmithKline), Asthma survey (Boehringer Ingelheim) and Arietta (Roche). Barry Make has participated in research studies and/ or served on medical advisory boards for AstraZeneca, Boehringer-Ingelheim, CSL Bering, GlaxoSmithKline, Forest, Novartis, Spiration, and Sunovion. In the past three years, Edwin K. Silverman received honoraria and consulting fees from Merck, grant support and consulting fees from GlaxoSmithKline and honoraria and travel support from Novartis. 


\section{References}

1. Sandler M. Is the lung a 'target organ' in diabetes mellitus? Arch Int Med. 1990; 150(7): 1385-1388.

doi: http://dx.doi.org/10.1001/archinte.1990.00390190051006

2. Mirrakhimov A. Chronic obstructive pulmonary disease and glucose metabolism: a bitter sweet symphony. Cardiovasc Diabetol. 2012; 11(1).

doi: http://dx.doi.org/10.1186/1475-2840-11-132

3. Sandler M, Bunn AE, Stewart RI, Cross-section study of pulmonary function in patients with insulin-dependent diabetes mellitus. Am Rev Respir Dis. 1987; 135(1): 223-229.

4. Klein OL, Meltzer P, Carnethon M, Krishnan JA. Type II diabetes mellitus is associated with decreased measures of lung function in a clinical setting. Respir Med. 2011; 105(7): 1095-1098. doi: http://dx.doi.org/10.1016/j.rmed.2011.03.010

5. van den Borst B, Gosker HR, Zeegers MP, Schols AMWJ. Pulmonary function in diabetes: A metaanalysis. Chest. 2010; 138(2): 393-406. doi: http://dx.doi.org/10.1378/chest.09-2622

6. Ford ES, Mannino DM. Prospective association between lung function and the incidence of diabetes: findings from the National Health and Nutrition Examination Survey Epidemiologic Follow-up Study. Diabetes Care. 2004; 27(12): 2966-70. doi: http://dx.doi.org/10.2337/diacare.27.12.2966

7. Eriksson KF, Lindgarde F Poor physical fitness, and impaired early insulin response but late hyperinsulinaemia, as predictors of NIDDM in middle-aged Swedish men. Diabetologia. 1996. 39(5): 573-579. doi: http://dx.doi.org/10.1007/BF00403304

8. Engstrom G, Janzon L. Risk of developing diabetes is inversely related to lung function: a population-based cohort study. Diabet Med. 2002; 19(2): 167-170.

doi: http://dx.doi.org/10.1046/j.1464-5491.2002.00652.x

9. Barrett-Connor E, Frette C. NIDDM, impaired glucose tolerance, and pulmonary function in older adults. The Rancho Bernardo Study. Diabetes Care. 1996; 19(12): 1441-1444.

doi: http://dx.doi.org/10.2337/diacare.19.12.1441

10. Bauer TA, Reusch JEB, Levi M, Regensteiner JG. Skeletal muscle deoxygenation after the onset of moderate exercise suggests slowed microvascular blood flow kinetics in type 2 diabetes. Diabetes Care. 2007; 30(11): 2880-2885.

doi: http://dx.doi.org/10.2337/dc07-0843

11. Kinney GL, Black-Shinn JL, Wan ES, et al. Pulmonary function reduction in diabetes with and without chronic obstructive pulmonary disease. Diabetes Care. 2014; 37(2):389-395. doi: http://dx.doi.org/10.2337/dc13-1435

12. Kalsi KK, Baker EH, Fraser O, et al. Glucose homeostasis across human airway epithelial cell monolayers: role of diffusion, transport and metabolism. Pflügers Arch. 2009; 457(5): 10611070. doi: http://dx.doi.org/10.1007/s00424-008-0576-4
13. Baker EH, Wood DM, Brennan, AL, Clark N, Baines DL, Phillips BJ. Hyperglycaemia and pulmonary infection. Proc Nutr Soc. 2006; 65(3): 227-35. doi: http://dx.doi.org/10.1079/PNS2006499

14. Baker EH, Bell D. Blood glucose: of emerging importance in COPD exacerbations. Thorax. 2009; 64(10): 830-832. doi: http://dx.doi.org/10.1136/thx.2009.118638

15. Kornum, JB, Thomsen RW, Riis A, Lervang H-H, Schǿnheyder HC, Srensen HT. Diabetes, glycemic control, and risk of hospitalization with pneumonia: a population-based casecontrol study. Diabetes Care. 2008; 31(8):1541-1545.

doi: http://dx.doi.org/10.2337/dc08-0138

16. Baker EH, Baines DL, Wood DM, Jones PW. Hyperglycaemia is associated with poor outcomes in patients admitted to hospital with acute exacerbations of chronic obstructive pulmonary disease. Thorax. 2006; 61(4): 284-289.

doi: http://dx.doi.org/10.1136/thx.2005.051029

17. Ilyas R, Wallis R, Soilleux EJ. High glucose disrupts oligosaccharide recognition function via competitive inhibition: a potential mechanism for immune dysregulation in diabetes mellitus. Immunobiology. 2011; 216(1-2): 126-131. doi: http://dx.doi.org/10.1016/j.imbio.2010.06.002

18. Reading PC, Allison J, Crouch EC, Anders EM. Increased susceptibility of diabetic mice to influenza virus infection: compromise of collectin-mediated host defense of the lung by glucose? J Virol. 1998; 72(8): 6884-6887.

19. Klein OL, Smith W, Tipping M, Peng J, Williams MV. Reduced diffusion lung capacity in patients with type 2 diabetes mellitus predicts hospitalization for pneumonia. Diabetes Res Clin Pract. 2011; 92(1): e12-15.

doi: http://dx.doi.org/10.1016/j.diabres.2010.12.012

20. Wannamethee SG, Shaper AG, Perry IJ. Smoking as a modifiable risk factor for type 2 diabetes in middle-aged men. Diabetes Care. 2001; 24(9): 1590-1595.

doi: http://dx.doi.org/10.2337/diacare.24.9.1590

21. Wannamethee SG, Shaper AG, Whincup PH. Modifiable lifestyle factors and the metabolic syndrome in older men: Effects of lifestyle changes. J Am Geriatr Soc. 2006; 54(12): 1909-1914. doi: http://dx.doi.org/10.1111/j.1532-5415.2006.00974.x

22. Regan EA, Hokanson JE, Murphy JR, et al. Genetic epidemiology of COPD (COPDGene) study design. COPD. 2010;7(1): 32-43. doi: http://dx.doi.org/10.3109/15412550903499522

23. Global initiative for chronic Obstructive Pulmonary Disease (GOLD). Global initiative for chronic Obstructive Disease: Global Strategy for the Diagnosis, Management, and Prevention of Chronic Obstructive Pulmonary Disease, updated November, 2006. GOLD website. http://wwwgoldcopdorg/uploads/users/ files/GOLDReport2006_0122pdf. Published 2006. Accessed July 23, 2016. 
24. Stewart JI, Moyle S, Crxiner GJ, et al. Automated telecommunication to obtain longitudinal follow-up in a multicenter cross-sectional COPD study. COPD. 2012; 9(5): 466472. doi: http://dx.doi.org/10.3109/15412555.2012.690010

25. Miller MR, Crapo R, Hankinson J, et al. General considerations for lung function testing. Eur Respir J. 2005; 26(1): 153-161. doi: http://dx.doi.org/10.1183/09031936.05.00034505

26. Hankinson JL, Odencrantz JR, Fedan KB. Spirometric reference values from a sample of the general U.S. population. Am J Respir Crit Care Med. 1999; 159(1): 179-187.

doi: http://dx.doi.org/10.1164/ajrccm.159.1.9712108

27. Jones PW, Quirk FH, Baveystock CM, Littlejohns P. A selfcomplete measure of health status for chronic airflow limitation. The St. George's Respiratory Questionnaire. Am Rev Respir Dis. 1992; 14(6): 1321-1327.

doi: http://dx.doi.org/10.1164/ajrccm/145.6.1321

28. Wan ES, Castaldi PJ, Cho MH, et al. Epidemiology, genetics, and subtyping of preserved ratio impaired spirometry (PRISm) in COPDGene. Respir Res. 2014; 15: 89.

doi: http://dx.doi.org/10.1186/s12931-014-0089-y

29. Fernandez-Real JM, Valdes S, Manco M, et al. Surfactant protein $\mathrm{d}$, a marker of lung innate immunity, is positively associated with insulin sensitivity. Diabetes Care. 2010; 33(4): 847-53. doi: http://dx.doi.org/10.2337/dc09-0542

30. More JM, et al. Smoking reduces surfactant protein D and phospholipids in patients with and without chronic obstructive pulmonary disease. BMC Pulm Med. 2010; 10: 53. doi: http://dx.doi.org/10.1186/1471-2466-10-53

31. Shakoori TA, et al. Serum surfactant protein D during acute exacerbations of chronic obstructive pulmonary disease. Dis Markers. 2009; 27(6): 287-294.

doi: http://dx.doi.org/10.1155/2009/759304

32. Herr C, Shaykhiev R, Bals R. The role of cathelicidin and defensins in pulmonary inflammatory diseases. Expert Opin Biol Ther. 2007; 7(9): 1449-61.

doi: http://dx.doi.org/10.1517/14712598.7.9.1449

33. Kiselar JG, et al. Modification of beta-defensin-2 by dicarbonyls methylglyoxal and glyoxal inhibits antibacterial and chemotactic function in vitro. PLoS One. 2015;10(8): e0130533.

doi: http://dx.doi.org/10.1371/journal.pone.0130533

34. Hamilton EJ, Martin N, Makepeace A, Sillars BA, Davis WA, Davis TME. Incidence and predictors of hospitalization for bacterial infection in community-based patients with type 2 diabetes: the fremantle diabetes study. PLoS One. 2013; 8(3): e60502. doi: http://dx.doi.org/10.1371/journal.pone.0060502
35. Mikkelsen KH, Knop Fk, Frost M, Hallas J, Pottegård A. Use of antibiotics and risk of type 2 diabetes: a population-based casecontrol study. J Clin Endocrinol Metab. 2015: c20152696. doi: http://dx.doi.org/10.1210/jc.2015-2696 